A. Pramesh Rao, G. Swarup and Gopal-Krishna, eds.

\title{
Detection of New Emission Components in PSR B0329+54
}

\author{
R. T. Gangadhara
}

Indian Institute of Astrophysics, Bangalore 560034, India

Y. Gupta

National Centre for Radio Astrophysics, TIFR, Post Bag 3, Ganeshkhind, Pune 411007, India

D. R. Lorimer

Arecibo Observatory, HC3 Box 53995, Arecibo, PR 00612, USA

\begin{abstract}
To study the structure of emission beam, we have analysed the single pulse data of PSR B0329+54 at 325 and $606 \mathrm{MHz}$. In order to unambiguously detect the weak emission components, we have developed a new data analysis technique, which we term "window-thresholding". By applying this technique to the data, we have detected three new emission components, and also confirmed the presence of a component which was proposed earlier. Hence our analysis indicates that PSR B0329+54 has nine components, which is among the highest of all the known pulsars. The symmetric distribution of pulse components about the pulse centre, indicates that the emission beam is conal.
\end{abstract}

\section{Data Analysis}

For the strong pulsar PSR B0329+54, the high-quality single pulses are easily observable. We obtained the data at $325 \mathrm{MHz}$ in March 1999 using the Giant Metrewave Radio Telescope near Pune, and the data at $606 \mathrm{MHz}$ was obtained in August 1996 using the 76-m Lovell Telescope at Jodrell Bank. For the analysis, we considered about 2500 single pulses at each frequency. The time resolutions of data at 325 and $606 \mathrm{MHz}$ were 0.516 and $0.250 \mathrm{~ms}$, respectively.

To estimate the pulse profiles which clearly show the presence of weaker components we developed a 'window-threshold' technique. In this technique, we set a window in longitude and employ an intensity threshold while considering the single pulses for making average profiles, i.e. we consider all those pulses, which have intensity levels above the threshold within the window. For threshold the $r m s$ in intensity was computed from the off-pulse region. In Figs. 1a\&b we have plotted the average pulse profiles which were obtained by applying this technique to nine component windows. The average profile obtained from all those pulses is plotted in Figs. 1c\&d, which clearly shows the presence of 9 emission components (I, II, III, IV, V, VI, VII, VIII, IX) in PSR B0329+54. 

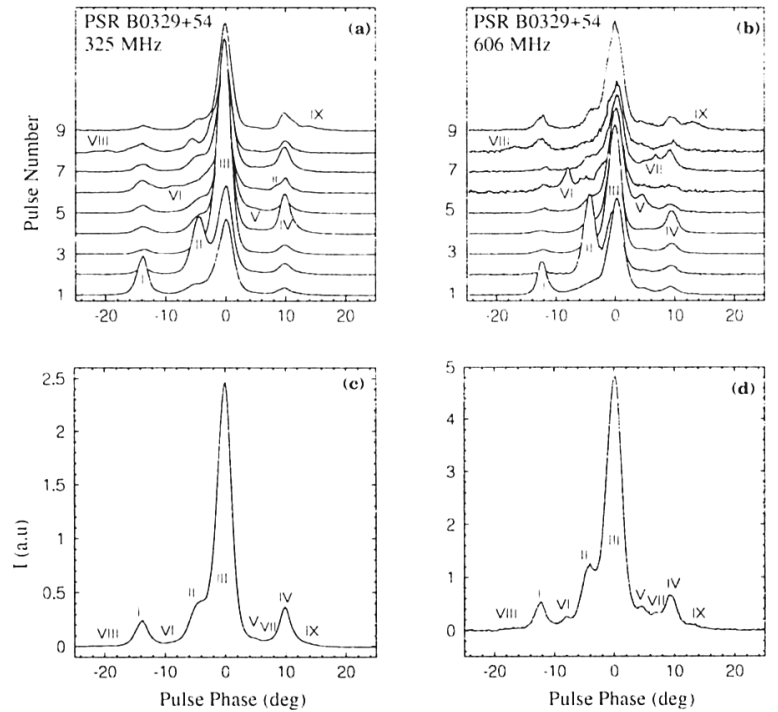

Figure 1. (a)\&(b) are the average profiles obtained by using the window-threshold technique for each component at 325 and $606 \mathrm{MHz}$, while (c)\&(d) are their average profiles with arbitrary units (a.u).

The components on trailing side of the profile are closely spaced compared to the leading side.

\section{Conclusion}

We have developed a technique based on windowing and thresholding, to detect the weak emission components in pulsar profiles. By applying it to the single pulse data of PSR B0329+54 we have detected three new emission components (VII, VIII and IX) of this pulsar, and also confirmed the presence of a component (VI) proposed by Kuzmin \& Izvekova (1996). The near-symmetric distribution of components around the core/centre of the profile, favours the idea that the pulsar emission beam is annular or conal (Oster \& Sieber 1977; Rankin 1983).

Acknowledgments. We thank A. G. Lyne for providing the Jodrell Bank data, and J. M. Rankin for useful discussions.

\section{References}

Kuzmin, A. D., \& Izvekova, V. A. 1996, PASP, Vol. 105, Pulsars: Problems \& Progress, ed. S. Johnston, M.A. Walker \& M. Bailes (San Francisco: ASP), 217

Oster, L., \& Sieber, W. 1977, ApJ, 58, 303

Rankin, J. M. 1983, ApJ, 274, 333

Previously published in "Pulsar Astronomy - 2000 and Beyond", ASP Conf. Series, 202, 163, 2000, Eds. Kramer, Wex \& Wielebinski 